\title{
Working with unaccompanied asylum-seeking young people: cultural considerations and acceptability of a cognitive behavioural group approach
}

\author{
Dorothy King ${ }^{1}$ and Glorianne Said ${ }^{2}$ \\ ${ }^{1}$ Doctorate in Clinical Psychology, Department of Psychology, Royal Holloway University of London, \\ Egham, Surrey TW20 OEX \\ ${ }^{2}$ Research Department of Clinical, Educational and Health Psychology, University College London, \\ 1-19 Torrington Place, London WC1E 7HB
}

Received 29 March 2018; Accepted 20 October 2018

\begin{abstract}
This paper outlines a psychological skills group for unaccompanied asylum-seeking young people with a focus on cultural adaptations in the context of a UK mental health service. Unaccompanied asylum-seeking young people have typically experienced multiple losses, traumatic experiences, significant disruption and psychosocial stressors. These experiences occur during a key developmental period and outside of the context of a supportive family environment. Mental health difficulties are estimated to be present in 41-69\% of this population. Prevalence rates are higher than among children seeking asylum with their families or children who are not from refugee or asylum-seeking backgrounds. Cognitive behavioural approaches were considered to be applicable and useful when working with this client group. Group approaches may offer unique benefits for this population through peer support and normalization. The group described was planned around three key themes: physical health needs, emotional wellbeing and resilience-building. A number of adaptations were made to meet the needs of this population which included engagement, considering physical health needs, sleep, language needs, issues related to power, race and status, and thinking about the needs of the group as young people. Attendance ratings, session rating scale outcomes, preliminary effectiveness data and qualitative feedback from young people identified that this is an acceptable approach for these young people. Unaccompanied asylumseeking young people require a broad package of care; however, making adaptations to routine practice allowed access to evidence-based interventions to support mental health and wellbeing.
\end{abstract}

Key words: children and adolescents, cognitive behaviour therapy, cultural adaptation, cross-cultural, trauma, young people

\section{Introduction}

A total of 25,670 unaccompanied asylum-seeking children (UASC) were registered within the European Union (EU) in 2017 and this number has increased significantly over the last 5 years (Eurostat, 2018). The term UASC is used to refer to children and young people under the age 
of 18 who are outside their country of origin, separated from both parents, are not being cared for by an adult who by law or custom has responsibility to do so, and who have applied for asylum in their own right (Home Office, 2016).

Children and young people become separated from their families and are forced to leave their country of origin for a number of reasons, including fear or experience of persecution, torture, violence, war and trafficking (Kohli and Mather, 2003). As a result, UASC are likely to have experienced multiple losses, have had limited or no periods of stability, and have often endured trauma in their country of origin as well as on the journey away from their home country, which can take many months (Fazel and Stein, 2002). Disruption within their country of origin and during their journey means that many young people have not had access to formal education or healthcare (Fazel et al., 2012). These experiences occur during a key developmental period (Blakemore, 2012; Konrad et al. 2013).

Within the refugee population, UASC are considered to have distinctive psychosocial needs and, following arrival in the receiving country, are required to navigate a multitude of social systems relating to their legal status, housing, care needs, health and education. UASC do not have access to buffering social support from family members which other refugee children might have, resulting in demands and responsibilities above and beyond what is typically expected of a child. While settling in the receiving country young people might experience displacement, discrimination, social exclusion and an increased sense of isolation (Bronstein and Montgomery, 2011; Fazel and Stein, 2002). In addition, the asylum process is a significant aspect of settling in a receiving country. Although young people seeking refugee status are allowed to reside in a receiving country until the age of 18, UASC are required to begin the asylum process; they have to contend with processes including giving a detailed testimony, an extended asylum interview, the threat of being returned to their country of origin, and prolonged periods of uncertainty while waiting for a decision. This process and the associated unpredictability can lead to considerable anxiety for young people.

Prevalence estimates of mental health disorders in unaccompanied asylum-seeking children range from 41 to 69\% (Bronstein and Montgomery, 2011; Coyle et al., 2016; Fazel and Stein, 2002; Jakobsen et al., 2014). UASC often present with disrupted sleep and feeding patterns, which can exacerbate emotional lability and distress (Polivy, 1996; Bronstein and Montgomery, 2013). The most frequent psychiatric diagnoses seen in refugee and asylumseeking children are post-traumatic stress disorder (PTSD), anxiety with sleep disorders, and depression; however, children can also present with a mixture of symptoms without meeting criteria for a single diagnostic category (Fazel and Stein, 2002). The prevalence of mental health difficulties in UASC are estimated to be higher than for children seeking asylum with their families and for children who are not from refugee or asylum-seeking backgrounds (Huemer et al., 2009) and is influenced by a number of individual, family, community and societal risk and protective factors (Fazel et al., 2012).

The mental health needs of UASC often relate to the consequences of significant exposure to multiple stressors and traumatic experiences during three different stages, as outlined above; while in their country of origin (pre-flight), during the journey to safety (flight) and when settling in the receiving country (post-flight; Yule, 2002). Although UASC have an increased level of vulnerability, adolescence presents a unique opportunity within which psychosocial support can play a key role with the potential to modify developmental trajectories (UNICEF, 2017). 
UASC may be unfamiliar with help seeking for any health need and the idea of psychological support may appear alien and confusing or, for some young people, threatening. Mental health needs are often interpreted differently within the cultures of origin for UASC and seeking support for mental health needs can be highly stigmatized. It is understood that there are a number of barriers for unaccompanied minors attending mental health services (Majumder et al., 2018). Initial assessment interviews for mental health services may also replicate interviews which UASC have to comply with as part of their asylum application, potentially making health appointments more aversive.

This paper outlines a psychological skills group developed for unaccompanied asylumseeking and refugee young people in the context of a mental health service in the UK, and describes specific considerations and adaptations made to meet the needs of this population. This paper includes a review of the acceptability and suitability of this approach using routine outcome monitoring, attendance data and feedback from young people.

\section{Psychosocial approaches with refugee children}

There is a growing evidence base for psychosocial interventions for children and young people from refugee and asylum-seeking backgrounds, which play a key role in addressing the needs of this population. Hobfoll et al. (2007) highlight five key principles for interventions for children and families who have been affected by war and mass violence: a sense of safety, calming, a sense of self-and-community efficacy, connectedness and hope.

Recent reviews highlight the multifaceted nature of the needs of refugee and asylumseeking children and adolescents and the importance of considering their wider context (Eruyar et al., 2017; Fazel and Betancourt, 2017). A number of key protective factors have been identified that support the psychological wellbeing of adolescent refugees, including social support networks and maintaining cultural links, which have led to suggestions that potential areas for intervention include friends and peers (Weine et al., 2014).

There is a need for cultural adaptations in interventions to address the diverse needs of children from refugee and asylum-seeking backgrounds (Fazel and Betancourt, 2017) and for those who have been exposed to traumatic events (Purgato et al., 2018). Interventions to address exposure to trauma have predominantly focused on PTSD (Fazel and Betancourt, 2017) with specific interventions such as Narrative Exposure Therapy (Schauer et al., 2011) demonstrating encouraging outcomes for refugees in high- and low-income countries (Neuner et al., 2004; Robjant and Fazel, 2010; Ruf et al., 2010). There is currently a mixed evidence base for other mental health interventions; however, there is an increasing focus on transdiagnostic treatments using common elements from evidence-based interventions, such as cognitive behavioural therapy, and including additional techniques aimed at establishing strengths, social-support building, or mind/body oriented skills (Fazel and Betancourt, 2017; Purgato et al., 2018).

Psychosocial approaches, including targeted interventions as well as group-based psychoeducation interventions, have been shown to be effective in reducing PTSD and functional impairment and in increasing hope, coping and social support in low-resource settings (Purgato et al., 2018) and in middle- and high-income countries (Ehntholt et al., 2005; Fazel and Betancourt, 2017; Sarkadi et al., 2018).

UNICEF (2017) highlights the acceptability of psychoeducation around stress to adolescents and that this can lead to significant reductions in stress hormone levels and 
symptoms of depression. Adolescence is a key developmental period with continued neural development and hormonal changes that interact with various psychological factors and changes in social contexts (Blakemore, 2012; Sawyer et al., 2012). This is considered to be a crucial time in which to intervene to support young people and interventions have the potential to improve mental health and wellbeing beyond adolescence (Patel et al., 2007; Sawyer et al., 2012; Laski, 2015; UNICEF, 2017).

\section{Applicability of cognitive behavioural approaches}

Cognitive behavioural approaches hold the basic understanding that different internal and external factors influence the development and maintenance of mental health difficulties and psychological distress. External factors include biopsychosocial influences in a person's history and current environment. Cognitive behavioural theory defines internal factors as emotions, physical sensations, thoughts and beliefs, and behaviours (Greenberger and Padesky, 1995). These factors have a reciprocal influence on each other and it is proposed that particular patterns of thinking and behaviour can lead to and maintain mental health difficulties and psychological distress. This also provides an avenue for intervention; change in one area is thought to influence change in others, and so supporting people to make helpful changes in one area can ameliorate symptoms of mental health difficulties and psychological distress. Therefore, providing coping strategies within the different areas of this model is likely to support wellbeing. The current group offered different interventions relating to thoughts, emotions, behaviours and physiological responses. There is an established evidence base for cognitive behavioural therapy (CBT) for children and adolescents presenting to mental health services (Graham and Reynolds, 2013) and encouraging evidence for supporting the psychological needs of child refugees in high income countries using a CBT approach (Ehntholt et al., 2005; Sarkadi et al., 2018).

Although not everyone who participates in this group may reach diagnostic thresholds for PTSD, due to the history of UASC, a trauma-informed approach was taken using ideas from trauma-focused therapeutic approaches. These were adapted from the stabilization phase of trauma-focused treatment including establishing a sense of safety, symptom management, and developing emotional awareness and emotion regulation skills (McFetridge et al., 2017). This was deemed to be important due to the instability that many UASC experience upon arrival in the UK, for example in relation to their legal status and housing. A phased model of intervention and holistic approach to care has been recommended for refugee children and young people (Ehntholt and Yule, 2006; Murray et al., 2008).

The Skills Training in Affective and Interpersonal Regulation (STAIR) approach to trauma developed by Cloitre and colleagues (2002) highlights different components to emotion regulation, adapted from Dialectical Behaviour Therapy (DBT; Linehan, 1987, 1993). These include identifying and labelling emotions, managing emotions, distress tolerance, acceptance of feelings and experiencing positive emotions. Difficulties with emotion regulation have been shown to mediate the relationship between trauma exposure and PTSD and depression in refugees, suggesting the importance of focusing on emotion regulation within therapy (Nickerson et al., 2015). Furthermore, emotion management and social support have been identified as important resources that can facilitate protection against developing PTSD and in recovery (Charuvastra and Cloitre, 2008). Ideas from this approach were therefore utilized within the group. 
Another approach drawn on was Compassion Focused Therapy (CFT; Gilbert, 2009, 2014). One of the key aims of CFT is to help develop experiences of safeness and soothing through compassion (Gilbert, 2009, 2014). UASC report experiencing a high sense of current threat and instability, which follows a period of high threat during their pre-flight and flight experiences. Young people describe limited or no experiences of soothing and safeness during these times. Specific compassion-focused techniques were therefore employed to support young people in reducing their current sense of threat and in developing experiences of safety and soothing.

\section{Service context}

The intervention being described takes place within a statutory National Health Service (NHS) child and adolescent mental health service for refugees and asylum seekers. The service operates within an ecological model (Bronfenbrenner, 1992) making use of systemic therapeutic approaches, offering consultation and resilience-based approaches within community settings, alongside assessment and intervention for specific mental health difficulties. In 2017, $69 \%$ of the referrals to the service were for unaccompanied asylumseeking young people.

Within the service, there is a need for an intervention for unaccompanied young people who present with varied mental health needs, and where individual work may not be indicated due to factors including unstable social circumstances or reluctance to engage in individual or trauma-focused work. Fazel and Betancourt (2017) identify how group approaches may offer unique benefits for this population though peer support and normalization, as well as encouraging connections among young people. The idea of an open group for UASC to address individual wellbeing was implemented in the service from February 2017. The group described in this paper was implemented from November 2017. Prior to this current group, the group had been informed by liberation and empowerment approaches, which identified that young people valued forming part of a group. The group is open to individuals regardless of the language they speak and is described to young people as a way of supporting overall wellbeing within an informal environment with other young people from similar backgrounds.

\section{Group content}

The group covers a range of ongoing topics to address the wellbeing needs of the young people. This was informed by the current evidence base around supporting the mental health needs of UASC, cognitive behavioural approaches, and an understanding of UASC health needs (UASC Health, 2016, unpublished data). This included the mental health consequences of trauma sequalae and the socio-emotional needs of young people. The group content was also informed by resilience-building approaches and coping skills relevant to adjusting to a new social environment. This led to three key themes around which the content of the group was based: physical health needs, emotional wellbeing, and resilience-building and empowerment. An outline of the content covered within the group is given in Table 1.

\section{Group participants}

Young people are referred to the group by clinicians in the service. This is an open group so young people can join at any time. Fourteen young people have been referred to date; Table 2 
Table 1. Outline of the content of the group sessions

\begin{tabular}{ll}
\hline Theme & Content \\
\hline Physical health needs & The importance of physical health \\
& Sleep hygiene and psychoeducation \\
& Sleep guides \\
& Routines \\
& Yoga \\
\hline Emotional wellbeing & Values, goal setting \\
& Identifying emotions, emotions in the body \\
& The fight/flight/freeze response \\
& Function of emotions \\
& Managing overwhelming emotions: DBT-informed \\
self-soothing and distress tolerance & Cycles of avoidance \\
& Grounding and memories \\
& Three affect-regulation systems (CFT) \\
& Soothing rhythm breathing, safe place imagery \\
& Hot cross bun and stress \\
& Managing worries and thoughts \\
& Relaxation and mindfulness exercises \\
\hline Strengths \\
Problem-solving booths \\
London/home - getting used to somewhere new, transitions \\
Racism \\
Tree of Life \\
Something I'm proud of \\
Hopes \\
\hline
\end{tabular}

DBT, dialectical behaviour therapy; CFT, compassion focused therapy.

Table 2. Demographic information for young people taking part in the group

\begin{tabular}{llllclll}
\hline ID & Gender & $\begin{array}{l}\text { Age at } \\
\text { referral }\end{array}$ & $\begin{array}{l}\text { Joined } \\
\text { in week }\end{array}$ & $\begin{array}{l}\text { Length of time in } \\
\text { UK at referral }\end{array}$ & $\begin{array}{l}\text { Country of } \\
\text { origin }\end{array}$ & Language & Interpreter \\
\hline 01 & Male & 17 & 1 & 10 months & Afghanistan & Dari & No \\
02 & Male & 16 & 1 & 18 months & Afghanistan & Dari & No \\
03 & Male & 16 & 1 & 3 months & Afghanistan & Pashto & Yes \\
04 & Female & 17 & 1 & 13 months & Ethiopia & Amharic & Yes \\
05 & Male & 17 & 2 & 3 months & Sudan & Arabic & Yes \\
06 & Male & 16 & 2 & 3 months & Sudan & Arabic & Yes \\
07 & Male & 17 & 4 & 4 months & Sudan & Arabic & Yes \\
08 & Male & 17 & 6 & 7 months & Ethiopia & Amharic & Yes \\
09 & Male & 15 & 7 & 13 months & Afghanistan & Dari & Yes \\
10 & Male & 17 & 11 & 15 months & Somalia & Somali & Yes \\
11 & Male & 17 & 19 & 8 months & Sudan & Arabic & Yes \\
12 & Male & 17 & 19 & 4 months & Sudan & Arabic & Yes \\
13 & Male & 14 & 19 & 8 months & Afghanistan & Pashto & Yes \\
14 & Male & 16 & 20 & 2 months & Ethiopia & Oromo & Yes \\
\hline
\end{tabular}


offers a representation of the young people within the group and their respective cultural backgrounds. Three young people no longer attend the group due to varying individual circumstances; one had conflicting schedules with other commitments and felt that he no longer required mental health support, one also felt that he no longer required mental health support, and one decided to engage with individual rather than group support. Eleven young people therefore attend on a consistent basis.

The mean age at referral was 16.36 years $(\mathrm{SD}=0.93)$ and 13 of the 14 young people referred were male. Interpreters were used to support 12 of the 14 young people referred to the group.

\section{Adaptations}

There are a few documented adaptions of CBT for clients from refugee backgrounds. Hinton et al. (2012) noted that in a 14-week stabilization group for adults with PTSD, interventions specifically focused on different somatic symptoms relating to anxiety, irritability and stress. In a 6-week group intervention for child refugees with psychological difficulties in secondary school, Ehntholt and colleagues (2005) made use of sleep hygiene, grounding strategies, imagery, activity scheduling and EMDR (eye movement desensitization and reprocessing) techniques to support young people with intrusions, hyperarousal and poor sleep.

The needs of the group required adaptations on the basis of ethnicity, language, culture, literacy and developmental needs. Vygotsky's Zone of Proximal Development (Vygotsky, 1978) informed the process of adapting interventions to ensure that these were accessible for young people who were unused to attending formal healthcare appointments, who were new to the country and who had a different understanding of mental health. This supported the rationale for scaffolding the psychoeducation interventions (Wood et al., 1976); beginning with understanding clients' own frame of reference then building to more complex interventions, such as the role of thoughts and the function of emotions, as the group's understanding developed.

The coordinated management of meaning theory (Cronen et al., 1988; Cronen, 2001) helped to inform how young people may make sense of their identity, experiences and difficulties. This informed adaptations within this group to make cognitive behavioural therapy more accessible. As facilitators, it was helpful to consider our own differences within the group context; this was at times named and acknowledged in discussions. It was also considered helpful to maintain an open stance of curiosity and to present interventions as suggestions or 'one' approach. The language used by facilitators also aimed to be mindful of young people's concerns relating to stigma around having mental health difficulties (Majumder et al., 2018); for example, the word 'stress' was often used instead of more nuanced terms such as psychological distress. As many clients were practising Muslims, concepts such as evolution were not presented as a part of psychoeducation discussions due to the different perspectives about evolution within Islam (Dagher and BouJaoude, 1997; Guessoum, 2016). When introducing CBT approaches, it was acknowledged that different ideas and practices may be more or less helpful for different individuals within the group, as young people had different presenting difficulties and strengths.

\section{Beginning with physical health}

Common presenting concerns reported by young people at assessment in the service included a number of physical health complaints including poor sleep, difficulties concentrating, 
headaches, pain and stomach aches. There is evidence in the research literature that individuals from non-Western cultures may report physical health difficulties more readily than psychological difficulties (Hinton and Lewis-Fernandez, 2010). A deliberate decision was made to begin the group with a discussion of physical health needs. It was thought that clients would engage better with such content at the beginning of the group, on the basis of their presenting complaints and being less familiar with talking therapies. This incorporated psychoeducation sessions on the roles of diet, routine and the importance of physical health. We used the PLEASE MASTER skill informed from the DBT approach as a way of introducing this (Linehan, 2015): treat physical illness, balanced eating, avoid mood altering drugs, balanced sleep, exercise, achieve mastery.

\section{Sleep}

Sleep is considered a unique need among UASC as, in addition to this being a possible expression of trauma-related arousal and reactivity within PTSD, sleep difficulties are often a consequence of having to alter one's sleep patterns and survive on significantly reduced sleep whilst on the journey to the receiving country. A multi-pronged approach to sleep was employed and young people were supported through implementation in a two-stage approach. The first session involved psychoeducation around the science of sleep and the introduction of a sleep diary to document day- and night-time factors that may affect sleep. The second session involved reviewing diaries and introducing strategies to respond to difficulties falling asleep and difficulties staying asleep. This included grounding strategies, sleep hygiene strategies and a behavioural sleep guide that offered instructions on gradually changing sleep patterns. The intervention also involved providing practical resources such as night lights, soothing scents, and grounding images to orient young people to their current location following nightmares or dissociation. As young people often spoke about rumination and worry before bed time, worry dolls were included as a means of helping young people to externalize their worries overnight (UASC Health, 2016, unpublished data). Efforts were made to make the resources more accessible; diaries were made using pictures and response formats were constructed so that young people could simply mark onto the diary rather than write. Young peoples' sleep was continually reviewed as part of the group process and sleep difficulties appeared to persist as a consequence of difficulties relating to post-traumatic stress.

\section{Supporting engagement with the group}

Unaccompanied minors' relationship with services, as well as their relationship to help overall (Reder and Fredman, 1996) was addressed from the start of the intervention. Prior to attending the group, the facilitators met with each young person to assess their unique mental health needs as well as how they felt about attending a group and whether they had any concerns about this. Group and individual reviews were held approximately every quarter, to gather feedback on whether young people felt the group was meeting their needs and to ascertain whether there were any topics the young people hoped to cover in future group sessions. It is thought that this supported young people's engagement with the group and enabled the facilitators to pre-empt or respond to any potential difficulties. 
To determine what topics to prioritize within the group, strategies informed by Acceptance and Commitment Therapy (Hayes et al., 2006) were employed to support clients to name goals in line with their individual values. The young people were asked to think about someone they admire and were interviewed by the facilitators to support this discussion, how these people were remembered and considered within their community, and what it is they value about them. Young people were asked to explain the meaning of this specific value in their culture. These were written on the board and other young people were asked about this concept, its meaning and expression in their culture and language. These concepts were then transformed into individual goals for the young people within the group.

\section{Power, race, status, control over circumstances}

Issues related to power, racism and status within receiving countries are acknowledged to have an impact on refugee mental health (Kastrup, 2016). UASC often report having limited or no control over their circumstances and adult figures are often required to coordinate much of their care without consulting the young person. To respond to this when using a cognitive behavioural model, the group facilitators took an explicitly collaborative stance throughout the group. Young people were invited to give their perspectives and the difficulties around professionals in power in some circumstances were openly named. Anxiety relating to young people's asylum application was also discussed in the group. When introducing the Greenberger and Padesky (1995) CBT model to the group, here and now worries as a consequence of the asylum process was used as an illustration, as well as helpful or less helpful ways of responding to this.

Certain interventions within the group also took on a more explicit empowerment aim. One such example is the use of problem solving booths (Owls, 2017, unpublished data), which allows young people to share their skills to support one another as well with individuals with more ascribed power, such as clinicians. Race and gender were also named and acknowledged alongside the impact of the other social graces on understanding and expressing mental health needs (Burnham, 2012), as well as on the differences within the group and between the group and the facilitators.

Young people's circumstances were understood to have an impact on their quality of life and which may exacerbate their mental health difficulties (Carlson et al., 2012; Fazel and Betancourt, 2017; Fazel et al., 2012). Alongside the group intervention, young people received practical support with their different social needs; at times liaising with others in the professional network and reporting on young people's mental health needs as part of the asylum process. Information about additional support young people received alongside the group can be found in Table 5 .

\section{Language}

Limited spoken English was not a barrier to taking part in the group. Following the British Psychological Society's best practice guidance for working with interpreters (2017), a language needs analysis was carried out as part of the initial assessment for the group, allowing clients to state their preferred spoken language and dialect. Using interpreters enabled the provision of an equitable and accessible service, in line with 
National Institute for Health and Care Excellence (NICE) guidelines (2005) as well as best practice recommendations for working with refugees (Mind, 2009). To respond to the concerns regarding the ongoing cost of interpretation, an alternative model of organizing interpreter bookings was suggested by employing interpreters directly through the NHS Trust, eliminating costly agency administration fees.

In setting up the group rules it was agreed that everything that was said in the group is interpreted, even if it was something said between young people who speak the same language. Interpretation within the group required active facilitation, reminding young people to pause when talking to allow for interpretation and monitoring when young people wanted to speak. For this reason, it is thought necessary to have two clinical facilitators to effectively run this group.

Less content was planned for each group session to account for the increased amount of time required to support interpretation. The language used by facilitators was intentionally kept simple and as jargon free as possible to support reliable interpretation and prevent distilling of the CBT model. During activities in the group, written language was avoided as much as possible as some participants were unable to read, even in their preferred language. However, when words were put on paper, interpreters or young people were invited to add the relevant word in their own language.

Five interpreters were required to run the group. The selected interpreters were familiar with mental health provision in the service and were invited for a training session prior to the start of the group. During this session, preferences for interpretation style around clarity and explanation were discussed, along with discussions around boundaries with young people and translating within a group. Care was taken to support interpreters who frequently worked with the group. Although it was not possible to formally debrief every session due to limitations on booking times, feedback was given to interpreters when possible, including positive feedback and noting appreciation of their contribution. Client responses to interpreters were also observed, and when clients had difficulty with a specific interpreter this was acknowledged and a different interpreter was booked for future sessions.

\section{Needs of young people}

The final consideration was being mindful of the needs of the group as young people. Adaptations were made for the content to be more user-friendly and interesting and to be delivered in a manner that considered young people's developmental stage (Sauter et al., 2009; Stallard, 2013). The start of every session included warm-up activities such as young people telling a joke, highlighting a pleasant event from the previous week or something new that they learned, with the aim of creating an informal and friendly atmosphere.

Games were used to identify different processes and concepts within the group to support engagement and salience of the content. Topics such as avoidance were introduced with a movement game asking young people to stand up or sit down if they ever engaged in certain avoidance activities. Metaphors relating to stress were brought to life using a bucket and water which the young people held and filled until it overflowed. Concepts such as the fight-or-flight response and the dual representation theory of memory (Brewin et al., 1996) were acted out by facilitators in a caricatured way to make them engaging and memorable. See Fig. 1 for a summary of the adaptations made for the group. 


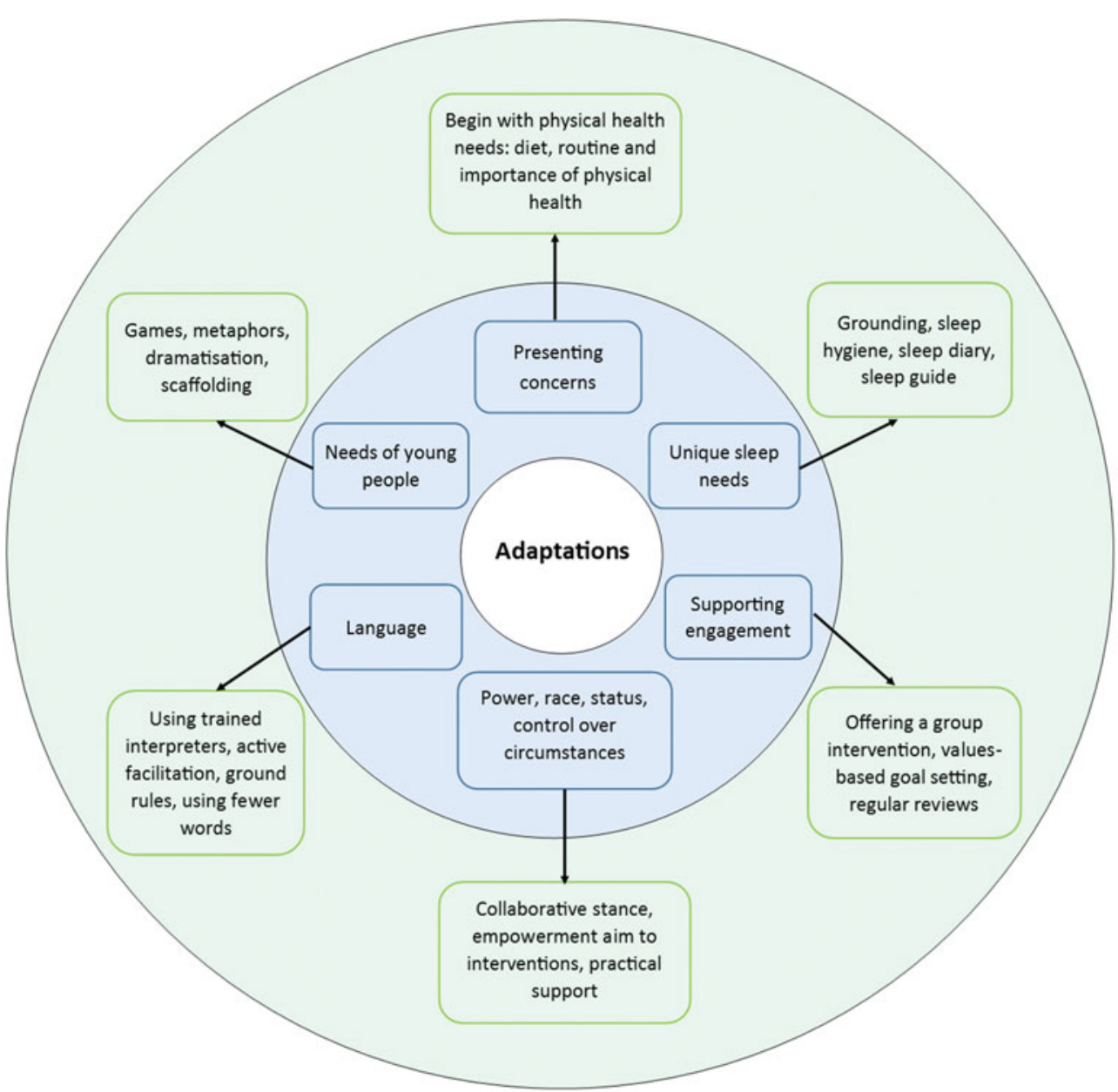

Figure 1. Adaptations made for the group

\section{Acceptability and feasibility of this approach}

The acceptability of this approach for UASC was evaluated using percentage attendance, review questions informed by the client change interview (Elliott, 1999) and session rating scales (Miller et al., 2000). The feasibility of this intervention was explored through reviewing the pragmatics of running such an intervention and preliminary effectiveness data.

\section{Acceptability}

The attendance was measured for every group session. Over the 35 weeks of the group included in this report, the mean attendance was $65 \%$ (SD = 18.04). Non-attendance reasons included conflicting appointments and social activities, withdrawal of funding for travel from the local authority, and additional requirements during the month of Ramadan. 
Table 3. Example quotes from young people taken from the group review session

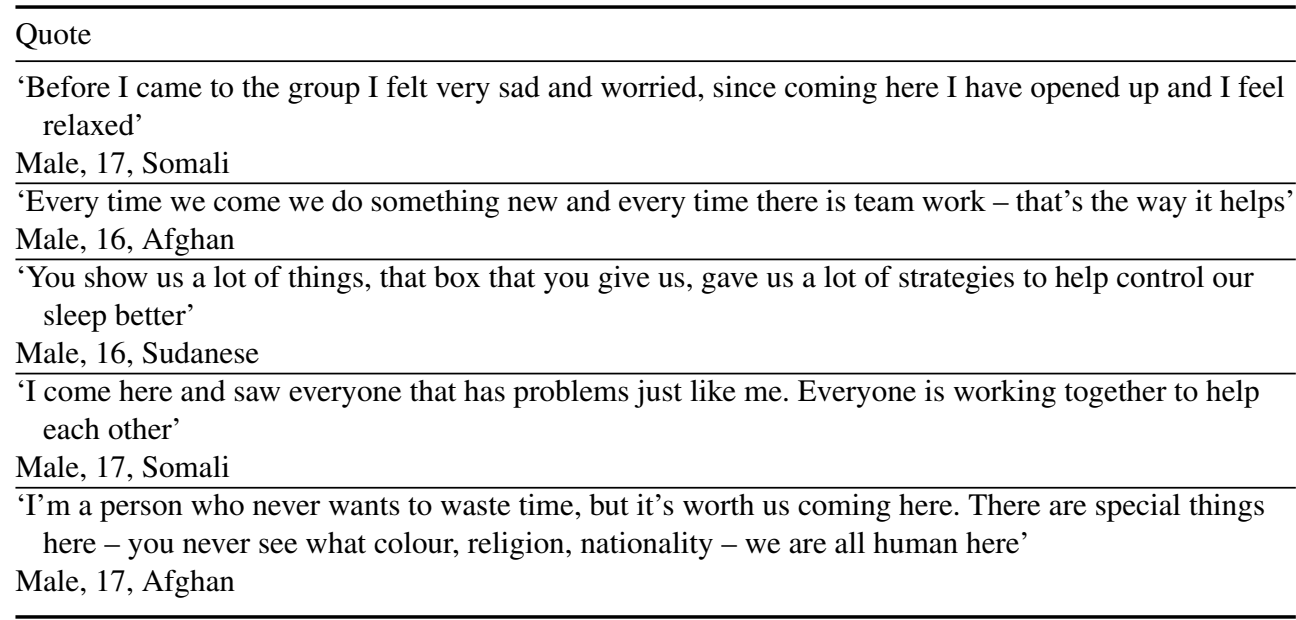

In a formal review session, group members were asked questions informed by the client change interview (Elliott, 1999) around how they were feeling, perceived helpfulness of the group, and any changes they may have noticed since the start of the group. All seven of the young people present during this session were able to identify and share ways in which they found the group helpful, what they valued about the group and suggestions for future content. Young people shared that they felt welcome and respected, valued being part of a group of young people in a similar position, learned a lot from the group and felt less stressed since joining. A few quotes from young people which offer an illustration of their feedback are given in Table 3.

Reviewing feedback from group members allowed us to evaluate whether the considerations made in planning and facilitating the group had been an acceptable method of providing mental health care for UASC within an NHS setting.

To monitor the suitability of the group and the adjustments made, Group Child Session Rating Scales (CGSRS) were used at the end of each session. This makes use of four visual analogue scales which prompts for ratings on listening, importance of topic, approach of content and overall approach to the group (Duncan et al., 2011). Practice recommendations for using the CGSRS include having open discussions when receiving feedback. This allowed for further flexibility in our approach and changes were made in subsequent groups in line with applicable feedback received at the end of each group session. The scales were measured and rated using a 12-point scale. Mean percentages for each category were calculated for ratings over 34 weeks of group feedback. The mean ratings on all four categories across 34 weeks were consistently over $90 \%$. A summary of the ratings for each category is provided in Table 4.

\section{Feasibility}

The practicalities relating to running an open group for unaccompanied minors alongside interpreters was considered in the feasibility of this intervention. Within this service, the group 
Table 4. Summary of ratings on the Session Rating Scale

\begin{tabular}{lll}
\hline Scale & Mean percentage rating & SD \\
\hline Listening & 97 & 4.73 \\
Importance of topic & 95 & 6.03 \\
Content & 96 & 6.57 \\
Overall approach & 94 & 7.75 \\
\hline
\end{tabular}

$\mathrm{SD}$, standard deviation.

was facilitated by final year trainee clinical psychologists, who were not directly employed by the trust, which minimized the cost of the intervention. Interpreter costs were also minimized through sharing interpreters. One interpreter was booked per language requirement, meaning that, at times, up to five young people only required one interpreter. It is also thought to be more cost effective to run a group intervention which young people attended rather than offering several individual appointments which unaccompanied minors may not attend due to mistrust of formal appointments and adults in positions of authority (Mind, 2009; Majumder et al., 2018). A group approach is also indicated to reduce social isolation, which is understood to prevent exacerbation of mental health difficulties (Fazel and Betancourt, 2017) that may require more complex interventions in the future (Cloitre et al., 2012).

\section{Clinical outcomes}

The Strengths and Difficulties Questionnaire (SDQ; Goodman, 1997, 2001) was used to monitor clinical outcomes. This is a measure routinely used across child and adolescent mental health services and within child settings such as schools and social care settings (Wolpert et al., 2015). Symptoms of PTSD were monitored using the Child Revised Impact of Events Scale 8-item version (CRIES-8; Perrin et al., 2005); however, this was completed at later stages in the group and was not used to monitor group outcomes.

The reliable change index (RCI; Jacobson and Truax, 1991) was used to determine whether change in scores between pre-intervention and a review at 35 weeks was deemed to be a statistically reliable change, beyond that which could be accounted for by measurement error. It was also reviewed whether scores crossed the clinical threshold, as recommended by Wolpert and colleagues (2015). Effect size using Cohen's $d$ (Cohen, 1988) was calculated for SDQ data for comparison of the change in scores from baseline to review.

Using normative data for the SDQ (Meltzer et al., 2000), it was calculated that statistically reliable change at the 5\% significance level was indicated by a score change greater than 6.32 . Of the eight people for whom paired data were available, four showed a reliable improvement in scores on the SDQ. One person was just below the statistically reliable threshold for improvement. No young people showed a deterioration; see Table 5.

The clinical threshold for identifying the presence of a psychiatric disorder for the selfreport SDQ is a score of $\geq 18$ (Goodman, 1997, 2001). At baseline, four of the young people for whom data were available $(N=10)$ scored above the clinical threshold on the SDQ. At the review time point, one of the young people for whom data were available $(N=12)$ scored above the clinical threshold; see Table 5. 
Table 5. Outcome measure data, number and percentage of sessions attended by each young person, and additional support and treatment received alongside the group

\begin{tabular}{|c|c|c|c|c|c|c|c|c|}
\hline \multirow[b]{2}{*}{ ID } & \multirow{2}{*}{$\begin{array}{l}\text { Number of } \\
\text { sessions attended } \\
(\% \text { attendance* })\end{array}$} & \multicolumn{3}{|c|}{ SDQ data } & \multicolumn{3}{|c|}{ CRIES-8 data } & \multirow{2}{*}{$\begin{array}{l}\text { Support and } \\
\text { treatment received } \\
\text { alongside the group }\end{array}$} \\
\hline & & Baseline & Review & $\begin{array}{l}\text { Reliable } \\
\text { improvement }\end{array}$ & Baseline & Review & $\begin{array}{l}\text { Reliable } \\
\text { improvement }\end{array}$ & \\
\hline 01 & $23(66)$ & 16 & 13 & No & & 30 & & Case management \\
\hline$\overline{02}$ & $16(46)$ & 22 & 21 & No & & 28 & & $\begin{array}{l}\text { Case management } \\
\text { Individual support }\end{array}$ \\
\hline$\overline{03}$ & $3(50)$ & 28 & & & & & & $\begin{array}{l}\text { Case management } \\
\text { Psychology-informed professional } \\
\text { opinion within asylum process }\end{array}$ \\
\hline$\overline{04}$ & $4(57)$ & 16 & & & & & & $\begin{array}{l}\text { Case management } \\
\text { Individual support }\end{array}$ \\
\hline$\overline{05}$ & $23(68)$ & 21 & 13 & Yes & & 1 & & $\begin{array}{l}\text { Case management } \\
\text { Psychology-informed professional } \\
\text { opinion within asylum process } \\
\text { Narrative Exposure Therapy }\end{array}$ \\
\hline 06 & $28(82)$ & 9 & 2 & Yes & 36 & 13 & Yes & $\begin{array}{l}\text { Case management } \\
\text { Psychology-informed professional } \\
\text { opinion within asylum process } \\
\text { Narrative Exposure Therapy }\end{array}$ \\
\hline$\overline{07}$ & $13(42)$ & 19 & 6 & Yes & 26 & 32 & No & $\begin{array}{l}\text { Case management } \\
\text { Psychology-informed professional } \\
\text { opinion within asylum process }\end{array}$ \\
\hline
\end{tabular}


Table 5. Continued

\begin{tabular}{|c|c|c|c|c|c|c|c|c|}
\hline \multirow[b]{2}{*}{ ID } & \multirow{2}{*}{$\begin{array}{l}\text { Number of } \\
\text { sessions attended } \\
\left(\% \text { attendance }{ }^{*}\right)\end{array}$} & \multicolumn{3}{|c|}{ SDQ data } & \multicolumn{3}{|c|}{ CRIES-8 data } & \multirow{2}{*}{$\begin{array}{l}\text { Support and } \\
\text { treatment received } \\
\text { alongside the group }\end{array}$} \\
\hline & & Baseline & Review & $\begin{array}{l}\text { Reliable } \\
\text { improvement }\end{array}$ & Baseline & Review & $\begin{array}{l}\text { Reliable } \\
\text { improvement }\end{array}$ & \\
\hline 08 & $17(59)$ & 14 & 8 & No & & 14 & & $\begin{array}{l}\text { Case management } \\
\text { Short-term individual psychology: } \\
\text { managing nightmares and CBT } \\
\text { for panic }\end{array}$ \\
\hline 09 & $3(30)$ & 7 & 6 & No & & & & Case management \\
\hline 10 & $15(63)$ & 13 & 4 & Yes & 29 & 14 & Yes & $\begin{array}{l}\text { Case management } \\
\text { Psychology-informed professional } \\
\text { opinion within asylum process }\end{array}$ \\
\hline 11 & $9(56)$ & & 12 & & & 24 & & $\begin{array}{l}\text { Case management } \\
\text { Psychology-informed professional } \\
\text { opinion within asylum process }\end{array}$ \\
\hline 12 & $12(75)$ & & 7 & & 23 & 25 & No & $\begin{array}{l}\text { Case management } \\
\text { Psychology-informed professional } \\
\text { opinion within asylum process }\end{array}$ \\
\hline 13 & $10(63)$ & & 15 & & 34 & 32 & No & $\begin{array}{l}\text { Case management } \\
\text { Individual grounding and } \\
\text { stabilisation } \\
\end{array}$ \\
\hline 14 & $14(93)$ & & 5 & & 36 & 24 & No & $\begin{array}{l}\text { Case management } \\
\text { Psychology-informed professional } \\
\text { opinion within asylum process }\end{array}$ \\
\hline Mean (SD) & & $16.50(6.28)$ & $9.33(5.50)$ & & $30.67(5.50)$ & $23.60(7.46)$ & & \\
\hline
\end{tabular}

${ }^{*}$ Individual percentage attendance is calculated based on the number of possible sessions the young person could have attended taking into account the week they started and ended the group. 'Completed the Child PTSD Symptom Scale (CPSS-V) instead of the CRIES-8.

Statistically reliable change on the SDQ has been calculated to be indicated by a score change of greater than 6.32 . Statistically reliable change on the CRIES-8 has been calculated to be indicated by a score change of greater than 13.03 . 
Cohen's $d$ for SDQ data was calculated to be 1.08, representing a large effect size (Cohen, 1988).

A discrepancy was identified between the total difficulties scores for young people on the SDQ and the difficulties identified on the CRIES-8. At baseline, of the six young people who completed the CRIES-8, all scored above the clinical cut-off of 17 (Perrin et al., 2005). One young person completed the Child PTSD Symptom Scale for DSM-5 (CPSS-V; Foa et al., 2018) instead of the CRIES-8 and scored 59 at baseline, which is above the clinical cut-off score of 31 (Foa et al., 2018). At the review time point, seven of the young people for whom data were available $(N=10)$ scored above the clinical threshold on the CRIES-8; see Table 5.

\section{Discussion}

This write up has attempted to describe how CBT approaches can be employed to meaningfully benefit UASC as part of a broader care package to support their mental wellbeing. In planning this group as clinical psychology doctoral students in the United Kingdom, many different skills garnered throughout our training were applicable. The content of the group was similar to that of other psychological skills groups that may exist in routine services; however, the manner in which these have been offered and delivered have been adjusted to ensure that they were applicable and helpful for UASC. Awareness of the evidence base, knowledge of the needs of UASC and the different difficulties that were prevalent for individuals in the group allowed us to put together an informed group plan to support young people's mental health needs.

UASC require a broad package of care. Being flexible in the manner with which care is delivered, allowing young people the opportunity to connect with others in a similar situation, and staging interventions to accommodate UASC biopsychosocial needs can support young people to engage with services which were previously unfamiliar. Responding to client needs and aiding with practical issues and the asylum process also provided the opportunity to shape young people's relationship with health services, particularly as larger institutions may be perceived as threatening (Majumder et al., 2018). Throughout the 35 weeks of the group, we have been struck by the young people's engagement. Once young people started attending the group, the return attendance rates have been very consistent. This, along with the session-bysession data outlined above, informs our understanding that the adaptations and the content of the group are meaningful and useful for UASC.

In our experience, the group has served a number of different functions. For young people who were reluctant to engage in more formal therapeutic provision, the group allowed UASC to receive empirically grounded support for a range of emotional needs while having their mental health needs reviewed as appropriate. Unlike other groups described in the literature or available in routine settings, eligibility is not limited to having a formal diagnosis. This has allowed the facilitators to consider UASC's wide-ranging needs and begin to offer psychoeducation regarding distressing symptoms, such as those characteristically associated with PTSD. This group may also be considered the first part of a phased approach for supporting complicated presentations (Cloitre et al., 2012).

The value of running this group as an open group is also considered significant. For young people whose social circumstances were quite chaotic, or for whom there are multiple demands on their time due to competing appointments, a time-limited intervention could have meant that young people could not access a significant proportion of what was offered. An 
open group also served as a place where young people could link in for further support and young people in this group reported valuing the accessibility of this format. Practically, young people were asked to notify facilitators via text message when they were unable to attend in order to cancel the interpreter booking, and this was frequently done. While this group included mostly new content every week, it is thought running the group in a modular format could make this more sustainable.

This paper has been able to identify preliminary effectiveness data for this approach using routine outcome measures. Unfortunately, outcome data was at times incomplete, which is reflective of some of the challenges of using measures to evaluate outcomes within routine CAMHS practice (Wolpert and Rutter, 2018). The SDQ, while not intended to identify potential PTSD symptoms, is largely used in child settings and may not be sensitive or specific enough to the needs of this client group (Stolk et al., 2017). This was identified by the proportion of young people in this group scoring below the clinical threshold on the SDQ while scoring significantly above the clinical cut-off for PTSD symptoms on the CRIES-8 or CPSS-V. Using a measure for global difficulties such as the Young Person's CORE (Twigg et al., 2009) may better capture specific needs within this client group including sleep difficulties, distressing thoughts and feelings and the perceived ability to reach out for support. This measure is being implemented more frequently with unaccompanied minors (The Children's Society, 2018). A specific measure to screen for symptoms of PTSD and for signs of distress within refugee populations, such as the CRIES and the Refugee Health Screener-15 (Hollifield et al., 2013) is considered to be a useful addition as young people may be unlikely to voluntarily report these difficulties.

While the trauma histories of UASC can at times be overwhelming, having the opportunity to be part of this group has allowed us to witness the many strengths, resilience and compassion that these young people possess. Slight adaptations to traditional practice allowed us to provide empirically grounded interventions to UASC at a developmental turning point and support young people's mental health, which has the potential to prevent the development of future difficulties.

\section{Main points}

(1) Unaccompanied asylum-seeking young people present with distinct psychosocial needs and there is a need for evidence-based interventions to support their mental health needs, delivered in a manner that is acceptable and meaningful for young people. It is crucial that interventions consider the wider context of young people, draw on protective factors and consider the existing evidence base alongside cultural adaptations in order to meet the needs of this population. Adolescence is a key time in which to intervene, and intervening at this stage has the potential to support mental health and wellbeing into adulthood.

(2) This paper described a number of adaptations made in routine clinical practice which allowed a cognitive behavioural informed group intervention to be delivered in a way that is acceptable to unaccompanied asylum-seeking young people. This group was delivered in the context of routine care in an outpatient mental health service in the UK and was feasible to implement in this setting. Return attendance rates were high and young people valued both the content of the group and the opportunity to meet other young people in similar situations. As facilitators, we felt privileged to work with these young people and witness their resilience and compassion, saw the importance of taking a flexible approach, 
and valued the opportunity to adapt our existing skills and knowledge to meet the needs of this population in a meaningful way.

\section{Acknowledgements}

We would like to thank the young people who have participated in this group for their enthusiasm, dedication and compassion during the group sessions as well as their support to write this paper. We would also like to thank Ms Katie Wilson and Ms Yaman Al Qadri, Masters students on placement in the service, who greatly supported the delivery of this project.

\section{Ethical statements}

The authors have abided by the Ethical Principles of Psychologists and Code of Conduct as set out by the American Psychological Association. Ethical approval was not required for this paper as it was not deemed to be research, as defined by the UK Policy Framework for Health and Social Care Research. However, written consent was sought from all young people who took part in the group for the inclusion of their anonymized demographic information and quotes in this paper.

\section{Conflicts of interest}

Dorothy King and Glorianne Said have no conflicts of interest with respect to this publication.

\section{Financial support}

No financial support was provided for this group or the write up of this paper.

\section{Suggestions for follow-up reading}

Betancourt TS, Meyers-Ohki SE, Charrow AP, Tol WA (2013). Interventions for children affected by war: an ecological perspective on psychosocial support and mental health care. Harvard Review of Psychiatry 21, 70-91.

British Psychological Society (2017). Working with Interpreters: Guidelines for Psychologists. UK: The British Psychological Society.

Ehntholt KA, Smith PA, Yule W (2005). School-based cognitive-behavioural therapy group intervention for refugee children who have experienced war-related trauma. Clinical Child Psychology and Psychiatry 10, 235-250.

Ehntholt KA, Yule W (2006). Practitioner review: assessment and treatment of refugee children and adolescents who have experienced war-related trauma. Journal of Child Psychology and Psychiatry 47, 1197-1210.

Eruyar S, Huemer J, Vostanis P (2017). How should child mental health services respond to the refugee crisis? Child and Adolescent Mental Health. Published online 7 November 2017: https://doi.org/10.1111/camh.12252

Fazel M, Betancourt TS (2017). Preventive mental health interventions for refugee children and adolescents in high-income settings. The Lancet Child and Adolescent Health 2, 121-132. 
Grey N, Young Y (2008). Cognitive behaviour therapy with refugees and asylum seekers experiencing traumatic stress symptoms. Behavioural and Cognitive Psychotherapy 36, 3-19.

Hinton DE, Rivera EI, Hofmann SG, Barlow DH, Otto MW (2012). Adapting CBT for traumatized refugees and ethnic minority patients: examples from culturally adapted CBT (CA-CBT). Transcultural Psychiatry 49, 340-365.

Measham T, Guzder J, Rousseau C, Pacione L, Blais-McPherson M, Nadeau L (2014). Refugee children and their families: supporting psychological well-being and positive adaptation following migration. Current Problems in Pediatric and Adolescent Health Care 44, 208-215.

Purgato M, Gross AL, Betancourt TS, Bolton P, Bonetto C, Gastaldon C, Gordon J, O'Callaghan P, Papola D, Peltonen K, Punamaki R-L, Richards J, Staples JK, Unterhitzenberger J, van Ommeren M, de Jong J, Jordans MJD, Tol WA, Barbui C (2018). Focused psychosocial interventions for children in low-resource humanitarian settings: a systematic review and individual participant data meta-analysis. Lancet Global Health 6, e390-400.

Sarkadi A, Ådahl K, Stenvall E, Ssegonja R, Batti H, Gavra P, Fängström K, Salari R (2018). Teaching tecovery techniques: evaluation of a group intervention for unaccompanied refugee minors with symptoms of PTSD in Sweden. European Child and Adolescent Psychiatry 27, 467-479.

Schauer M, Neuner F, Elbert T (2017). Narrative Exposure Therapy for Children and Adolescents (KIDNET). In Evidence-Based Treatments for Trauma Related Disorders in Children and Adolescents (ed. MA Landolt, M Cloitre, U Schnyder), pp. 227-250. Switzerland: Springer International Publishing.

Smith P, Dyregrov A, Yule W, Perrin S, Gjestad R, Gupta L (2000). Children and War: Teaching Recovery Techniques. Bergen, Norway: Foundation for Children and War.

UNICEF Office of Research - Innocenti (2017). The Adolescent Brain: A Second Window of Opportunity. Florence: UNICEF Office of Research - Innocenti.

\section{References}

Blakemore S-J (2012). Imaging brain development: the adolescent brain. Neuroimage 61, 397-406.

Brewin CR, Dalgleish T, Joseph S (1996). A dual representation theory of posttraumatic stress disorder. Psychological Review 103, 670.

British Psychological Society (2017). Working with Interpreters: Guidelines for Psychologists. UK: The British Psychological Society.

Bronfenbrenner U (1992). Ecological systems theory. In Six Theories of Child Development: Revised Formulations and Current Issues (ed. R Vasta), pp. 187-249. London, UK: Jessica Kingsley Publishers.

Bronstein I, Montgomery P (2011). Psychological distress in refugee children: a systematic review. Clinical Child and Family Psychology Review 14, 44-56.

Bronstein I, Montgomery P (2013). Sleeping patterns of Afghan unaccompanied asylum-seeking adolescents: a large observational study. PLoS One 8, e56156.

Burnham J (2012). Developments in Social GRRRAAACCEEESSS: Visible-Invisible and VoicedUnvoiced. In Culture and Reflexivity in Systemic Psychotherapy: Mutual Perspectives (ed. IB Krause), pp. 139-160. London: Karnac.

Carlson BE, Cacciatore J, Klimek B (2012). A risk and resilience perspective on unaccompanied refugee minors. Social Work 57, 259-269.

Charuvastra A, Cloitre M (2008). Social bonds and posttraumatic stress disorder. Annual Review of Psychology 59, 301-328.

Children's Society (2018). Distress Signals - Unaccompanied Young People's Struggle for Mental Health Care. London, UK: The Children's Society. 
Cloitre M, Courtois CA, Ford JD, Green BL, Alexander P, Briere J, Herman JL, Lanius R, Stolbach BC, Spinazzola J, Van der Kolk BA, Van der Hart O (2012). The ISTSS Expert Consensus Treatment Guidelines for Complex PTSD in Adults. International Society for Traumatic Stress Studies.

Cloitre M, Koenen KC, Cohen LR, Han H (2002). Skills training in affective and interpersonal regulation followed by exposure: a phase-based treatment for PTSD related to childhood abuse. Journal of Consulting and Clinical Psychology 70, 1067-1074.

Cohen J (1988). Statistical Power Analysis for the Behavioral Sciences (2nd edn). USA: Lawrence Erlbaum Associates.

Coyle R, Bowen S, Mullin S, Sayer N, Siggers G, Bennett S (2016). Physical and mental health needs of unaccompanied children seeking asylum: a descriptive analysis in Kent, UK. The Lancet $\mathbf{3 8 8}$, S40.

Cronen VE (2001). Practical theory, practical art, and the pragmatic-systemic account of inquiry. Communication Theory 11, 14-35.

Cronen VE, Chen V, Pearce WB (1988). Coordinated management of meaning: a critical theory. International and Intercultural Communication Annual 12, 66-98.

Dagher ZR, BouJaoude S (1997). Scientific views and religious beliefs of college students: the case of biological evolution. Journal of Research in Science Teaching 34, 429-445.

Duncan BL, Miller SD, Sparks JA, Murphy JJ (2011). Child Group Session Rating Scale (CGSRS).

Ehntholt KA, Smith PA, and Yule W (2005). School-based cognitive-behavioural therapy group intervention for refugee children who have experienced war-related trauma. Clinical Child Psychology and Psychiatry 10, 235-250.

Ehntholt KA, Yule W (2006). Practitioner review: assessment and treatment of refugee children and adolescents who have experienced war-related trauma. Journal of Child Psychology and Psychiatry 47, 1197-1210.

Elliott R (1999). Client change interview protocol. Network for Research on Experiential Therapies.

Eruyar S, Huemer J, Vostanis P (2017). Review: how should child mental health services respond to the refugee crisis? Child and Adolescent Mental Health. Published online 7 November 2017: https://doi.org/10.1111/camh.12252

Eurostat (2018). Asylum applicants considered to be unaccompanied minors by citizenship, age and sex - annual data. Retrieved from: http://appsso.eurostat.ec.europa.eu/nui/show.do? dataset=migr_asyunaa

Fazel M, Betancourt TS (2017). Preventive mental health interventions for refugee children and adolescents in high-income settings. The Lancet Child and Adolescent Health 2, 121-132.

Fazel M, Reed RV, Panter-Brick C, Stein A (2012). Mental health of displaced and refugee children resettled in high-income countries: risk and protective factors. The Lancet 379, 266-282.

Fazel M, Stein A (2002). The mental health of refugee children. Archives of Disease in Childhood $\mathbf{8 7}$, 366-370.

Foa EB, Asnaani A, Zang Y, Capaldi S, Yeh R (2018). Psychometrics of the Child PTSD Symptom Scale for DSM-5 for trauma-exposed children and adolescents. Journal of Clinical Child and Adolescent Psychology 47, 38-46.

Gilbert P (2009). Introducing compassion-focused therapy. Advances in Psychiatric Treatment 15, 199208.

Gilbert P (2014). The origins and nature of compassion focused therapy. British Journal of Clinical Psychology 53, 6-41.

Goodman R (1997). The Strengths and Difficulties Questionnaire: a research note. Journal of Child Psychology and Psychiatry 38, 581-586.

Goodman R (2001). Psychometric properties of the strengths and difficulties questionnaire. Journal of the American Academy of Child and Adolescent Psychiatry 40, 1337-1345. 
Graham P, Reynolds S (2013). Cognitive Behaviour Therapy for Children and Families (3rd edn). New York: Cambridge University Press.

Greenberger D, Padesky C (1995). Mind over Mood. New York: Guilford Press.

Guessoum N (2016). Islamic Theological Views on Darwinian Evolution. Retrieved from: http:// religion.oxfordre.com/view/10.1093/acrefore/9780199340378.001.0001/acrefore-9780199340378e-36

Hayes SC, Luoma JB, Bond FW, Masuda A, Lillis J (2006). Acceptance and commitment therapy: model, processes and outcomes. Behaviour Research and Therapy 44, 1-25.

Hinton DE, Lewis-Fernandez R (2010). Idioms of distress among trauma survivors: subtypes and clinical utility. Culture, Medicine and Psychiatry 34, 209-218.

Hobfoll SE, Watson P, Bell CC, Bryant RA, Brymer MJ, Friedman MJ et al. (2007). Five essential elements of immediate and mid-term mass trauma intervention: empirical evidence. Psychiatry 70, 283-315.

Hollifield M, Verbillis-Kolp S, Farmer B, Toolson EC, Woldehaimanot T, Yamazaki J, Holland A, St. Clair J, SooHoo J (2013). The Refugee Health Screener-15 (RHS-15): development and validation of an instrument for anxiety, depression, and PTSD in refugees. General Hospital Psychiatry 35, 202-209

Home Office (2016). Home Office Funding: Unaccompanied Asylum Seeking Children (UASC). UK: Home Office.

Huemer J, Karnik NS, Voelkl-Kernstock S, Granditsch E, Dervic K, Friedrich MH, Steiner H (2009). Mental health issues in unaccompanied refugee minors. Child and Adolescent Psychiatry and Mental Health 3, 13.

Jacobson NS, and Truax P (1991). Clinical significance: a statistical approach to defining meaningful change in psychotherapy research. Journal of Consulting and Clinical Psychology 59, 12.

Jakobsen M, Demott MA, Heir T (2014). Prevalence of psychiatric disorders among unaccompanied asylum-seeking adolescents in Norway. Clinical Practice and Epidemiology in Mental Health 10, 53-58.

Kastrup M (2016). The impact of racism and discrimination on mental health of refugees and asylum seekers. European Psychiatry 33, S43.

Kohli R, Mather R (2003). Promoting psychosocial well-being in unaccompanied asylum seeking young people in the United Kingdom. Child and Family Social Work 8, 201-212.

Konrad K, Firk C, Uhlhaas PJ (2013). Brain development during adolescence: neuroscientific insights into this developmental period. Deutsches Ärzteblatt International 110, 425.

Laski L (2015). Realising the health and wellbeing of adolescents. British Medical Journal 351, h4119. Published online 14 September 2015. doi: https://doi.org/10.1136/bmj.h4119

Linehan MM (1987). Dialectical behavior therapy for borderline personality disorder: theory and method. Bulletin of the Menninger Clinic 51, 261-276.

Linehan MM (1993). Skills Training Manual for Treating Borderline Personality Disorder. New York: Guilford Press.

Linehan MM (2015). DBT Skills Training Manual. New York: Guilford Press.

Majumder P, Vostanis P, Karim K, O'Reilly M (2018). Potential barriers in the therapeutic relationship in unaccompanied refugee minors in mental health. Journal of Mental Health 1-8.

McFetridge M, Swan AH, Heke S, Karatzias T, Greenberg N, Kitchiner N, Morley R (2017). Guideline for the Treatment and Planning of Services for Complex Post-Traumatic Stress Disorder in Adults. UK: UK Psychological Trauma Society.

Meltzer H, Gatward R, Goodman R, Ford F (2000) Mental Health of Children and Adolescents in Great Britain. London: The Stationery Office.

Miller SD, Duncan BL, Johnson LD (2000). The Session Rating Scale 3.0. Chicago, IL.

Mind (2009). A Civilised Society: Mental Health Provision for Refugees and Asylum-Seekers in England and Wales. London, UK: Mind. 
Murray LK, Cohen JA, Ellis BH, Mannarino A (2008). Cognitive behavioral therapy for symptoms of trauma and traumatic grief in refugee youth. Child and Adolescent Psychiatric Clinics of North America 17, 585-604

National Institute for Health and Care Excellence (2005). Post-Traumatic Stress Disorder: Management. Clinical guideline 26.

Neuner F, Schauer M, Klaschik C, Karunakara U, Elbert T (2004). A comparison of narrative exposure therapy, supportive counseling, and psychoeducation for treating posttraumatic stress disorder in an African refugee settlement. Journal of Consulting and Clinical Psychology 72, 579587.

Nickerson A, Bryant RA, Schnyder U, Schick M, Mueller J, Morina N (2015). Emotion dysregulation mediates the relationship between trauma exposure, post-migration living difficulties and psychological outcomes in traumatized refugees. Journal of Affective Disorders 173, 185-192.

Owls (2017). Problem Solving Booths. Retrieved from:https://www.problemsolvingbooths.com/

Patel V, Flisher AJ, Hetrick S, McGorry P (2007). Mental health of young people: a global publichealth challenge. The Lancet 369, 1302-1313.

Perrin S, Meiser-Stedman R, Smith P (2005). The Children's Revised Impact of Event Scale (CRIES): validity as a screening instrument for PTSD. Behavioural and Cognitive Psychotherapy 33, 487-498.

Polivy J (1996). Psychological consequences of food restriction. Journal of the American Dietetic Association 96, 589-592.

Purgato M, Gross AL, Betancourt TS, Bolton P, Bonetto C, Gastaldon C, Gordon J, O'Callaghan P, Papola D, Peltonen K, Punamaki R-L, Richards J, Staples JK, Unterhitzenberger J, van Ommeren M, de Jong J, Jordans MJD, Tol WA, Barbui C (2018). Focused psychosocial interventions for children in low-resource humanitarian settings: a systematic review and individual participant data meta-analysis. Lancet Global Health 6, e390-400.

Reder P, Fredman G (1996). The relationship to help: interacting beliefs about the treatment process. Clinical Child Psychology and Psychiatry 1, 457-467.

Robjant K, Fazel M (2010). The emerging evidence for narrative exposure therapy: a review. Clinical Psychology Review 30, 1030-1039.

Ruf M, Schauer M, Neuner F, Catani C, Schauer E, Elbert T (2010). Narrative exposure therapy for 7- to 16-year-olds: a randomized controlled trial with traumatized refugee children. Journal of Traumatic Stress 23, 437-445.

Sarkadi A, Ådahl K, Stenvall E, Ssegonja R, Batti H, Gavra P, Fängström K, Salari R (2018). Teaching recovery techniques: evaluation of a group intervention for unaccompanied refugee minors with symptoms of PTSD in Sweden. European Child and Adolescent Psychiatry 27, 467-479.

Sauter FM, Heyne D, Westenberg PM (2009). Cognitive behavior therapy for anxious adolescents: developmental influences on treatment design and delivery. Clinical Child and Family Psychology Review 12, 310-335.

Sawyer SM, Afifi RA, Bearinger LH, Blakemore S-J, Dick B, Ezeh AC, Patton GC (2012). Adolescence: a foundation for future health. The Lancet 379, 1630-1640.

Schauer M, Neuner F, Elbert T (2011). Narrative Exposure Therapy: A Short Term Treatment For Traumatic Stress Disorders (2nd edn). Cambridge, MA: Hogrefe Publishing.

Stallard P (2013). Adapting cognitive behaviour therapy for children and adolescents. In Cognitive Behaviour Therapy for Children and Families (3rd edn) (eds P Graham and S Reynolds), pp. 22-33. Cambridge UK: Cambridge University Press.

Stolk Y, Kaplan I, Szwarc J (2017). Review of the strengths and difficulties questionnaire translated into languages spoken by children and adolescents of refugee background. International Journal of Methods in Psychiatric Research 26 (4).

Twigg E, Barkham M, Bewick BM, Mulhern B, Connell J, Cooper M (2009). The Young Person's CORE: development of a brief outcome measure for young people. Counselling and Psychotherapy Research 9, 160-168. 
UASC Health (2016). Retrieved from: http://www.uaschealth.org

UNICEF Office of Research - Innocenti (2017). The Adolescent Brain: A Second Window of Opportunity. Florence: UNICEF Office of Research - Innocenti.

Vygotsky LS (1978). Mind in Society: The Development of Higher Psychological Processes. USA: Harvard University Press.

Weine SM, Ware N, Hakizimana L (2014). Fostering resilience: protective agents, resources, and mechanisms for adolescent refugees' psychosocial well-being. Adolescent Psychiatry 4, 164-176.

Wolpert M, Görzig A, Deighton J, Fugard AJ, Newman R, Ford T (2015). Comparison of indices of clinically meaningful change in child and adolescent mental health services: difference scores, reliable change, crossing clinical thresholds and 'added value'- an exploration using parent rated scores on the SDQ. Child and Adolescent Mental Health 20, 94-101.

Wolpert M, Rutter H (2018). Using flawed, uncertain, proximate and sparse (FUPS) data in the context of complexity: learning from the case of child mental health. BMC Medicine 16, 82.

Wood D, Bruner JS, Ross G (1976). The role of tutoring in problem solving. Journal of Child Psychology and Psychiatry 17, 89-100.

Yule W (2002). Alleviating the effects of war and displacement on children. Traumatology 8, 160.

\section{Learning objectives}

(1) To provide an overview of the psychosocial needs of unaccompanied asylumseeking minors.

(2) To discuss the relevance of cognitive behavioural therapeutic approaches to this client group.

(3) To describe culturally informed ways of working within an outpatient mental health setting.

(4) To review the acceptability of this approach for young people and the process as professionals. 